\title{
Article \\ Skeletal Muscle Metabolomic Responses to Endurance and Resistance Training in Rats under Chronic Unpredictable Mild Stress
}

\author{
Xiangyu Liu ${ }^{1,+}$, Xiong Xue ${ }^{1,+}$, Junsheng Tian ${ }^{2,3}$, Xuemei Qin ${ }^{2,3}$, Shi Zhou ${ }^{4} \mathbb{D}$, Anping Chen ${ }^{1, *}$ \\ and Yumei Han 1,3,*(D)
}

check for

updates

Citation: Liu, X.; Xue, X.; Tian, J.; Qin, X.; Zhou, S.; Chen, A.; Han, Y.

Skeletal Muscle Metabolomic

Responses to Endurance and

Resistance Training in Rats under Chronic Unpredictable Mild Stress. Int. J. Environ. Res. Public Health 2021, 18, 1645. https://doi.org/10.3390/ ijerph18041645

Academic Editors: Amelia Guadalupe Grau and Fraser Carson

Received: 30 November 2020

Accepted: 2 February 2021

Published: 9 February 2021

Publisher's Note: MDPI stays neutral with regard to jurisdictional claims in published maps and institutional affiliations.

Copyright: (C) 2021 by the authors Licensee MDPI, Basel, Switzerland This article is an open access article distributed under the terms and conditions of the Creative Commons Attribution (CC BY) license (https:// creativecommons.org/licenses/by/ $4.0 /)$
1 School of Physical Education, Shanxi University, Taiyuan 030006, China; lxy18834850353@163.com (X.L.); 15135117678@163.com (X.X.)

2 Modern Research Center for Traditional Chinese Medicine, Shanxi University, Taiyuan 030006, China; jstian@sxu.edu.cn (J.T.); qinxm@sxu.edu.cn (X.Q.)

3 The Institute for Biomedicine and Health, Shanxi University, Taiyuan 030006, China

4 School of Health and Human Sciences, Southern Cross University, Lismore, NSW 2480, Australia; Shi.Zhou@scu.edu.au

* Correspondence: sxchenanping@sxu.edu.cn (A.C.); hanyumei@sxu.edu.cn (Y.H.)

+ These authors have contributed equally to this work and are regarded as joint first authors.

\begin{abstract}
The objectives of this study were to compare the antidepressant effects between endurance and resistance exercise for optimizing interventions and examine the metabolomic changes in different types of skeletal muscles in response to the exercise, using a rat model of chronic unpredictable mild stress (CUMS)-induced depression. There were 32 male Sprague-Dawley rats randomly divided into a control group (C) and 3 experimental groups: CUMS control (D), endurance exercise (E), and resistance exercise (R). Group E underwent 30 min treadmill running, and group R performed 8 rounds of ladder climbing, 5 sessions per week for 4 weeks. Body weight, sucrose preference, and open field tests were performed pre and post the intervention period for changes in depressant symptoms, and the gastrocnemius and soleus muscles were sampled after the intervention for metabolomic analysis using the ${ }^{1} \mathrm{H}-\mathrm{NMR}$ technique. The results showed that both types of exercise effectively improved the depression-like symptoms, and the endurance exercise appeared to have a better effect. The levels of 10 metabolites from the gastrocnemius and 13 metabolites from the soleus of group D were found to be significantly different from that of group $C$, and both types of exercise had a callback effect on these metabolites, indicating that a number of metabolic pathways were involved in the depression and responded to the exercise interventions.
\end{abstract}

Keywords: ${ }^{1} \mathrm{H}-\mathrm{NMR}$; depression; exercise; skeletal muscle; metabolomics

\section{Introduction}

Depression is a common illness, with more than 264 million people affected by it globally [1]. The World Health Organization announced in January 2020 that depression is a leading cause of disability worldwide and a major contributor to the overall global burden of diseases [2]. The pathogenesis of depression has not been fully elucidated [3]. However, it has been evident that, as one aspect of its etiology, depression is closely related to metabolic disorders [4]. It has also been shown that physical exercise is a promising intervention for depression and has the advantages of lower cost, fewer side effects, and many other health benefits compared with pharmaceutical treatments [5-7]. At present, the research on the mechanisms of the antidepressant effects of exercise mainly focuses on the neurobiological changes [8-10], however, less attention is given to the potential peripheral mechanisms, such as metabolite changes in skeletal muscle.

Metabolomics is an emerging discipline that studies the overall change of metabolites in cells and the organism [11]. With metabolomics, we can explore the pattern of change 
in the endogenous metabolite spectrum of an organism under the influence of external environmental stimulation, gene mutation, and pathophysiological factors [12]. Skeletal muscle is a highly plastic tissue with a number of identified phenotypes and different types of exercise training, such as endurance vs. resistance training, resulting in specific responses and adaptations in the metabolic characteristics and regulatory pathways in different types of muscle fibers $[13,14]$. It is known that some muscles consist of predominantly slow-twitch, oxidative fibers, such as the soleus in rats, and some consist of predominantly fast-twitch, glycolytic fibers, such as the gastrocnemius, while most muscles have a mixture of fast and slow-twitch fibers [15]. Metabolites, as the end or by- products of cellular biochemical activities, have been studied to explore the relationships between genotypes and phenotypes, reflecting the regulatory effect of gene expression and protein changes on a biological system [16]. To date, most of the studies on exercise intervention for depression are on the effects of endurance training (mainly relying on aerobic energy metabolism) [17,18], although resistance training (relying on mixed anaerobic and aerobic metabolism depending on exercise intensity, duration, and repetition) has also been shown to be beneficial in the management of several health conditions, such as reducing obesity, improving sarcopenia, and so on $[19,20]$. It is important to explore the efficacy and understand the mechanisms of various types of exercise interventions for the prevention and treatment of depression as well as other types of health disorders. We have previously examined urine metabolomic responses to endurance and resistance training in rats under chronic unpredictable mild stress (CUMS) [21]. However, there has been no report to date that has examined the regulatory roles of skeletal muscle metabolites by using metabolomics in relation to the pathophysiological responses to exercise interventions for depression.

The objectives of this study were to compare the antidepressant effects between two types of exercise, endurance vs. resistance training, for optimizing interventions, and examine the metabolomic changes in the soleus and gastrocnemius muscles in response to the exercise, using the ${ }^{1} \mathrm{H}-\mathrm{NMR}$ metabolomics technology in combination with the multivariate statistical method, in a rat model of CUMS-induced depression. With consideration of the metabolic characteristics of different types of muscle fibers, as well as different types of exercise, it was hypothesized that the endurance and resistance exercise interventions would lead to different changes in metabolomics in the fast- and slow-twitch muscle fibers. The outcomes of the study would contribute to a better understanding of the therapeutic effects and their underlying mechanisms of exercise interventions for depression.

\section{Materials and Methods}

\subsection{Subjects}

Male Sprague-Dawley (SD) rats, SPF grade, with a body mass of 180 200 g, were obtained from Beijing Weitong Lihua Experimental Animal Technology Co., LTD (Animal License Number: SCXK, Beijing, China, 2016-0006). The rats were housed in an airconditioned room with an ambient temperature of $23 \pm 1.5^{\circ} \mathrm{C}$, humidity $45 \% \pm 15 \%$, and light $12 \mathrm{~h}$ on and $12 \mathrm{~h}$ off.

\subsection{Ethical Approval}

This study was approved by the Animal Experiments Ethics Committee of the University (approval number: SXULL2018006). The maximal effort was made to minimize animal suffering and the number of animals necessary for the acquisition of reliable data.

\subsection{Chronic Unpredictable Mild Stress (CUMS) Modeling and Grouping}

The method of modeling has been presented previously [21]. Briefly, 32 male SD rats were randomly divided into 4 groups, with 8 in each group: (1) Blank control group (C), (2) CUMS control group (D), (3) CUMS with endurance exercise group (E), and (4) CUMS with resistance exercise group (R). All rats in the experimental groups received CUMS modeling stress during the 1 st 4 weeks. The stimuli included: No water for $24 \mathrm{~h}$, fasting for 
$24 \mathrm{~h}$, tail clipping for $2 \mathrm{~min}, 4^{\circ} \mathrm{C}$ ice bath for $5 \mathrm{~min}$, ultrasonic stimulation for $3 \mathrm{~h}$, movement restraint for $3 \mathrm{~h}$, thermal stimulation for $10 \mathrm{~min}$, and electric shocks to feet (voltage $36 \mathrm{~V}$, shock interval $10 \mathrm{~s}$, for 10 times), day and night reversal (placed under fluorescent light for $24 \mathrm{~h}$ ). The rats were given only one type of stimulus each day. To prevent the rats from forming memories in response to stress, the same stress stimulus was not repeated for at least 7 days.

\subsection{Evaluation of Model Validity}

The validity of the modeling was evaluated by the body weight (BW), sucrose preference test (SPT), and open field test (OFT) to assess depression-like behavior at the end of each week.

\subsubsection{Body Weight Measurement}

The BW was measured between 3 p.m. and 5 p.m. on the last day of each week.

\subsubsection{Sucrose Preference Test}

Anhedonia is an important symptom of depression and can be routinely measured by sugar water preference [22]. Each rat (single-caged) was placed simultaneously with 2 water bottles-the 1 st $24 \mathrm{~h}$ with 2 bottles of $1 \%$ sucrose solution, and the 2 nd $24 \mathrm{~h}$ with 1 bottle of $1 \%$ sucrose solution and 1 bottle of plain drinking water. At the end of the training, the subjects were deprived of water and fasted for $12 \mathrm{~h}$, then subjected to a sucrose preference text by being given 2 pre-weighed solution vials: $100 \mathrm{~mL}$ of $1 \%$ sucrose solution and $100 \mathrm{~mL}$ of plain water. The 2 vials were placed at the same height. The sucrose preference rate was determined by the consumption of the $1 \%$ sucrose solution relative to the total liquid intake, measured after 3 h; i.e., sucrose preference rate $(\%)=$ sucrose intake $(\mathrm{g}) /$ total liquid intake $(\mathrm{g}) \times 100 \%$.

\subsubsection{Open Field Test}

The floor of the open-field experimental device $(100 \mathrm{~cm} \times 100 \mathrm{~cm})$ was divided into 25 equal squares. Rats were placed in the center square for $1 \mathrm{~min}$ to adapt to the environment. The number of crossings and standings in 5 min was recorded. A crossing referred to entering into a new square with 4 paws, reflecting the rat's ability to exercise. The standing referred to that the forelimbs of the rat raised from the ground, reflecting their ability to explore [23].

\subsection{Exercise Protocols}

\subsubsection{Endurance Exercise}

The endurance exercise consisted of $30 \mathrm{~min}$ running on a motor-driven treadmill, at zero degree slope and speed of $3 \mathrm{~m} / \mathrm{min}$ for the first $5 \mathrm{~min}, 5 \mathrm{~m} / \mathrm{min}$ for the next $5 \mathrm{~min}$, and $8 \mathrm{~m} / \mathrm{min}$ for the remaining $20 \mathrm{~min}$, with a total of $30 \mathrm{~min}$ per day, 5 days per week for 4 weeks.

\subsubsection{Resistance Exercise}

The resistance exercise group was given a familiarization training of $15 \mathrm{~min}$ a day for 3 days. They climbed a ladder of $1.1 \mathrm{~m}$ long, $0.18 \mathrm{~m}$ wide, with a $2 \mathrm{~cm}$ grid, and 80 degrees of inclination, 4 times on the 1st day, 6 times on the 2nd day, and 8 times on the 3rd day. In the formal training, each rat climbed 8 times a day, 5 times a week, for 4 weeks, with incremental loads. The load was added to the rat's tail by a lead sheet wrapped in Vega tape, with a weight of $25 \%$ of the animal's BW during the first week of training. The load was increased by $25 \%$ BW each week until $100 \%$ BW in the 4 th week. The animals that stopped climbing were touched on the back by a ruler or tweezers to encourage the movement. At the top of the ladder, there was a wooden box in which the rats could rest for $2 \mathrm{~min}$ before the commencement of the next climbing repetition. 


\subsection{Muscle Sample Collection and Preparation}

At the end of the 4 weeks of training, the same assessments were performed for BW, STP, and OFT (Figure 1). After overnight fasting, the gastrocnemius and soleus muscle samples were obtained under deep anesthesia. The samples were cleaned from blood and trimmed for connective tissues and stored at $-80^{\circ} \mathrm{C}$ before metabolomics analysis.

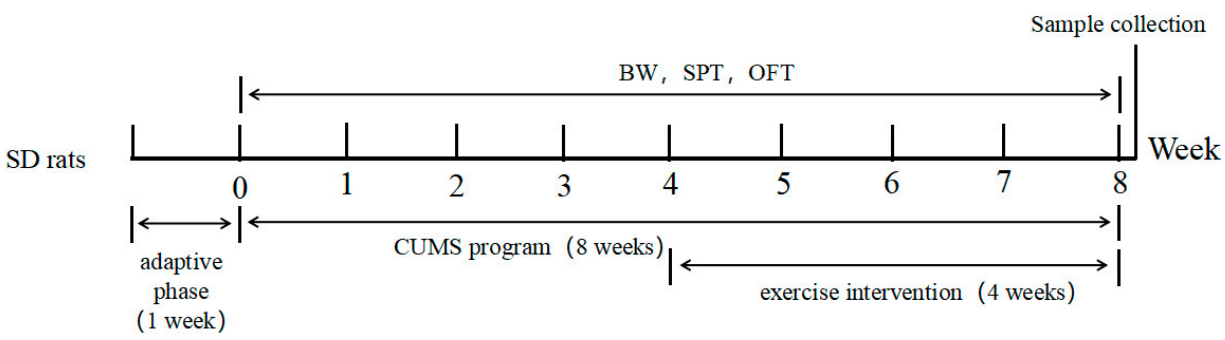

Figure 1. Experimental flow chart. BW, body weight; SPT, sucrose preference test; OFT, open field test.

\subsection{NMR Metabolomics}

\subsubsection{Sample Pretreatment}

After the muscle sample was thawed, $200 \mathrm{mg}$ of tissue was diced into small pieces and placed in a $5 \mathrm{~mL}$ Eppendorf (EP) tube, and $1 \mathrm{~mL}$ methanol-water $(v / v, 2: 1)$ was added. The homogenate was extracted twice on ice and transferred to a $2 \mathrm{~mL}$ centrifuge tube and centrifuged at $4{ }^{\circ} \mathrm{C}, 13,000 \mathrm{r} / \mathrm{min}$, for $15 \mathrm{~min}$. The supernatant was transferred to a $2 \mathrm{~mL}$ $\mathrm{EP}$ tube for drying with nitrogen blowing. After drying, phosphate buffer $(\mathrm{pH}=7.40)$ was added to $750 \mu \mathrm{L}$, and the sample was centrifuged at $4{ }^{\circ} \mathrm{C}, 13,000 \mathrm{r} / \mathrm{min}$, for $20 \mathrm{~min}$. Then $600 \mu \mathrm{L}$ of supernatant was transferred to a nuclear magnetic tube with diameter of $5 \mathrm{~mm}$ for testing.

\subsubsection{NMR Detection Conditions}

Bruker 600 MHz AVANCE III NMR spectrometer (Bruker Biospin, Rheinstetten, Germany) was used to scan the samples 64 times with the Noesygppr1d pulse sequence. The parameters were set to a spectral width of $8 \mathrm{kHz}$, a mixing time of $150 \mathrm{~ms}$, a relaxation delay of $320 \mathrm{~ms}$, a sampling point of $64 \mathrm{k}$, and an accumulation number of 64 . The pre-saturation method was used to suppress the water peak during the relaxation delay, the spectrometer bias was set at the water peak position, and the free induction decay signal was converted into a ${ }^{1} \mathrm{H}-\mathrm{NMR}$ spectrum by Fourier transformation.

\subsection{Statistical Analysis}

All data were expressed as the mean \pm standard deviation. SPSS statistical software (Version 18.0, SPSS Inc., Chicago, IL, USA) was used for statistical analysis. Graph Pad Prism software (Version 7.0, La Jolla, CA, USA) was used to generate graphs. Data from the behavioral tests were first checked for normal distribution and then analyzed by 2way ANOVA with repeated measures. One-way ANOVA was performed for differential metabolites. Principal component analysis (PCA), partial least squares-discriminant analysis (PLS-DA), and orthogonal partial least squares discriminant analysis (OPLS-DA) was performed using SIMCA-P multivariate statistical analysis software (Version 14.0, Umetrics, Umea, Sweden).

\section{Results}

\subsection{Behavioral Changes}

Behavioral indicators of each group were measured once a week during the experiment. As shown in Figure 2, after the four weeks of modeling, compared with group C, the BW, sucrose preference rate, and the number of crossings and standings were significantly lower in groups $\mathrm{D}, \mathrm{E}$, and $\mathrm{R}(p<0.05$ or $p<0.01)$, indicating that the CUMS-induced de- 
pression model was established successfully. After the four weeks of exercise intervention period, the $\mathrm{BW}$, the sucrose preference rate, and the numbers of crossing and standing of the groups D, E, and R were still significantly lower than that of group $C(p<0.05$ or $p<0.01)$. When comparing with group $\mathrm{D}$, the sucrose preference rate and the numbers of crossings and standing of group E were significantly increased $(p<0.05)$, and the number of crossings in group R was significantly increased $(p<0.05)$. (See Supplementary Materials for detailed data).
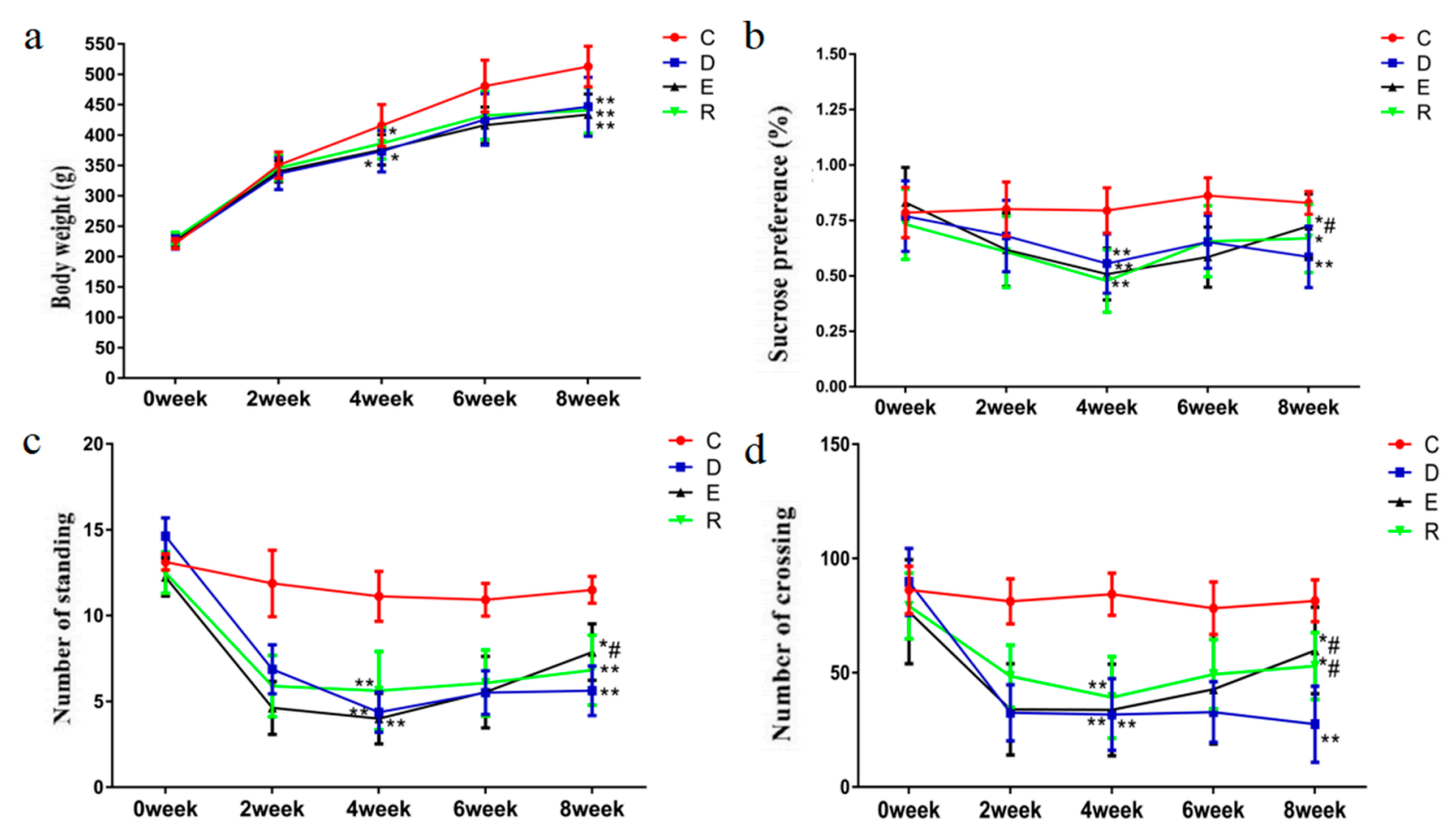

Figure 2. Effects of different exercise modes on behavioral indices of CUMS-induced depression rats. Comparison with group C: ${ }^{*} p<0.05,{ }^{* *} p<0.01$; comparison with group D: ${ }^{\#} p<0.05,{ }^{\# \#} p<0.01$; (a) body weight, (b) sucrose preference, (c) number of standing, (d) number of crossing.

\subsection{Skeletal Muscle Metabolomics}

\subsubsection{Skeletal Muscle NMR Spectrum}

A typical ${ }^{1} \mathrm{H}-\mathrm{NMR}$ spectrum of skeletal muscle in CUMS-induced depression rats was shown in Figure 3. Based on HMDB (the Human Metabolome Database, http:/ / www. hmdb.ca/ (accessed on 25 November 2020)), BMRB (Biological Magnetic Resonance Data Bank, http:/ / www.bmrb.wisc.edu / (accessed on 25 November 2020)), and other public databases, and consulting with related literature to identify the nuclear magnetic map of skeletal muscle, a total of 32 metabolites was identified from the gastrocnemius muscle (Figure 3a), and a total of 26 metabolites was identified from the soleus muscle (Figure 3b).

\subsubsection{Multivariate Statistical Analysis}

Multivariate statistical analysis was used to find out the differences among groups, to reflect more information on the map. Partial least square discriminant analysis (PLS-DA, Figures $4 \mathrm{a}$ and $5 \mathrm{a}$ ) and orthogonal partial least square discriminant analysis (OPLS-DA, Figures $4 \mathrm{c}$ and $5 \mathrm{c}$ ) were performed on muscle samples. It can be seen from Figures $4 \mathrm{a}$ and $5 \mathrm{a}$ that groups $C$ and $D$ were obviously separated, and $R 2$ and Q2 generated by the $Y$ variable were both smaller than the primary values (Figures $4 \mathrm{~b}$ and $5 \mathrm{~b}$ ), proving that the model was effective and reliable. Then, OPLS-DA analysis was performed on groups $C$ and D, VIP values were combined with S-plot (Figures $4 \mathrm{~d}$ and $5 \mathrm{~d}$ ). According to VIP $>1$ and $p<0.05$, a total of 10 metabolites with significant differences were detected in the gastrocnemius of depressed rats, and a total of 13 metabolites with significant differences was detected in the soleus. Then, the differential metabolites were analyzed by one-way ANOVA. 


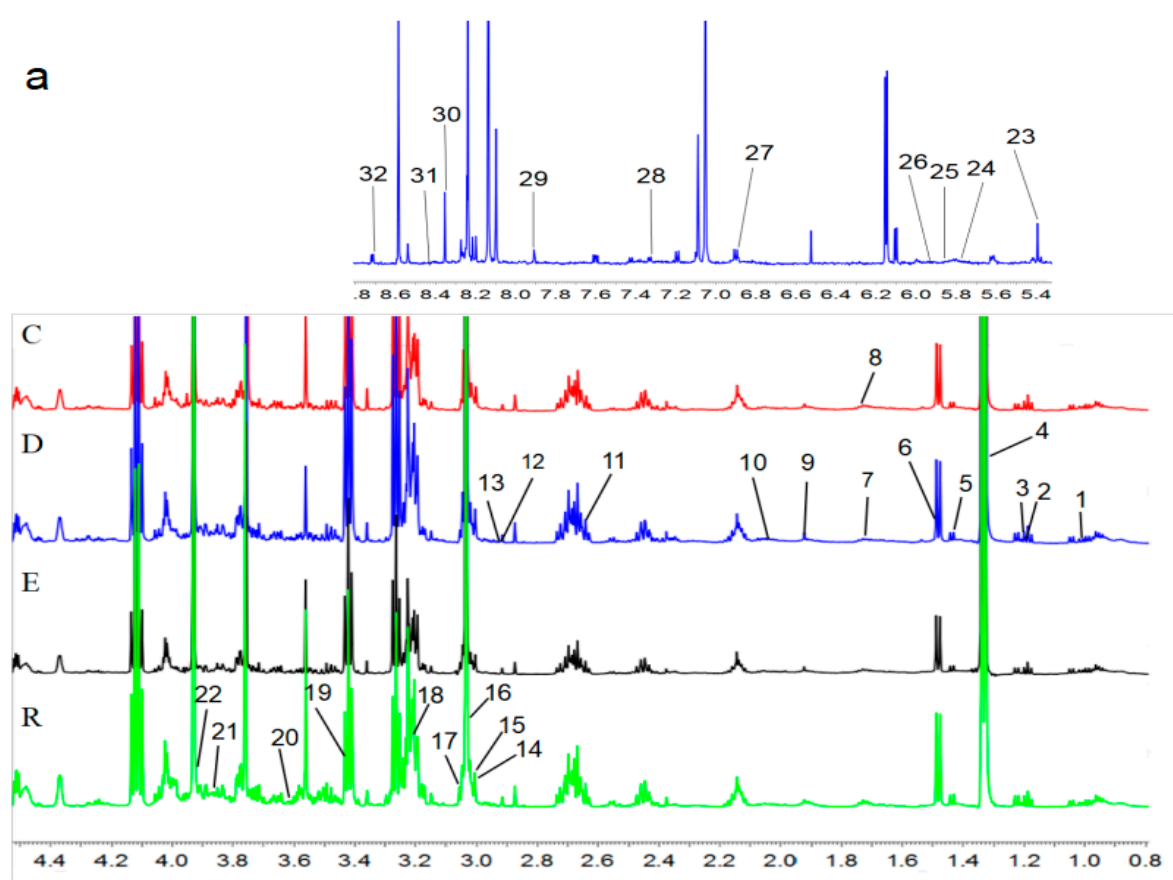

(ठ)

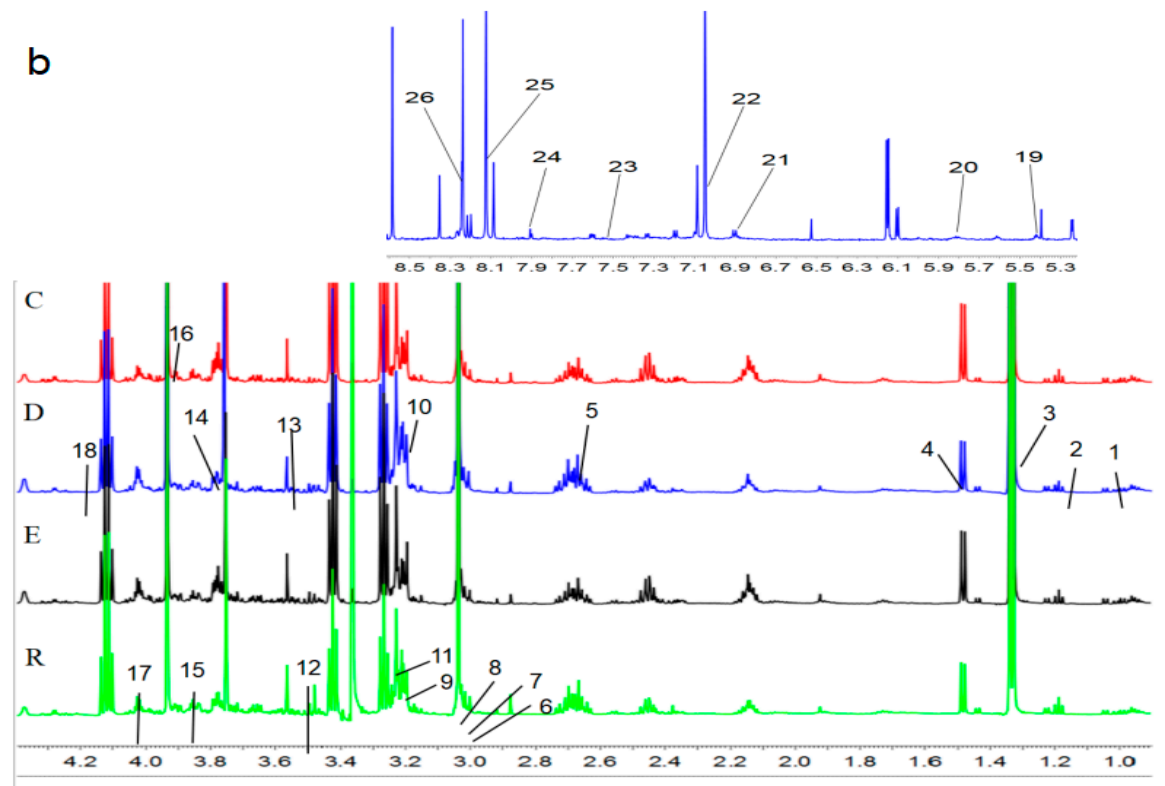

(ס)

Figure 3. ${ }^{1} \mathrm{H}-\mathrm{NMR}$ metabolite spectra $(\delta)$ of the gastrocnemius muscle (a) and soleus muscle (b). C, control group; D, CUMS control group; E, CUMS endurance exercise group; R, CUMS resistance exercise group. (a) (1) Isoleucine; (2) Propylene glycol; (3) very low-density lipoprotein; (4) Lactate; (5) Lysine; (6) Alanine; (7) Ornithine; (8) Acetate; (9) Ornithine acetate; (10) Glutamate; (11) Citric acid; (12) Dimethylglycine; (13) Dimethylamine; (14) $\gamma$-aminobutyric acid; (15) Anserine; (16) Creatinine; (17) Creatine; (18) Choline phosphate; (19) Taurine; (20) Threonine; (21) Alpha-glucose; (22) Betaine; (23) Allantoin; (24) Uracil; (25) Uridine; (26) Cytidine; (27) Tyrosine; (28) Phenylalanine; (29) Histidine; (30) Inosine; (31) Formate and (32) Nicotinamide. (b) (1) Leucine; (2) 3-Aminoisobutyrate; (3) Lactate; (4) Alanine; (5) N-acetylaspartate; (6) Aspartic acid; (7) Anserine; (8) Creatinine; (9) Choline; (10) Choline phosphate; (11) Glycerophosphate choline; (12) Glucose; (13) Glycine; (14) Glutamine; (15) Glycerol; (16) Alpha-glucose; (17) AMP; (18) Threonine; (19) Raffinose; (20) Uridine; (21) Tyrosine; (22) Histidine; (23) Hippurate; (24) Xanthine; (25) Hypoxanthine and (26) Inosine. 

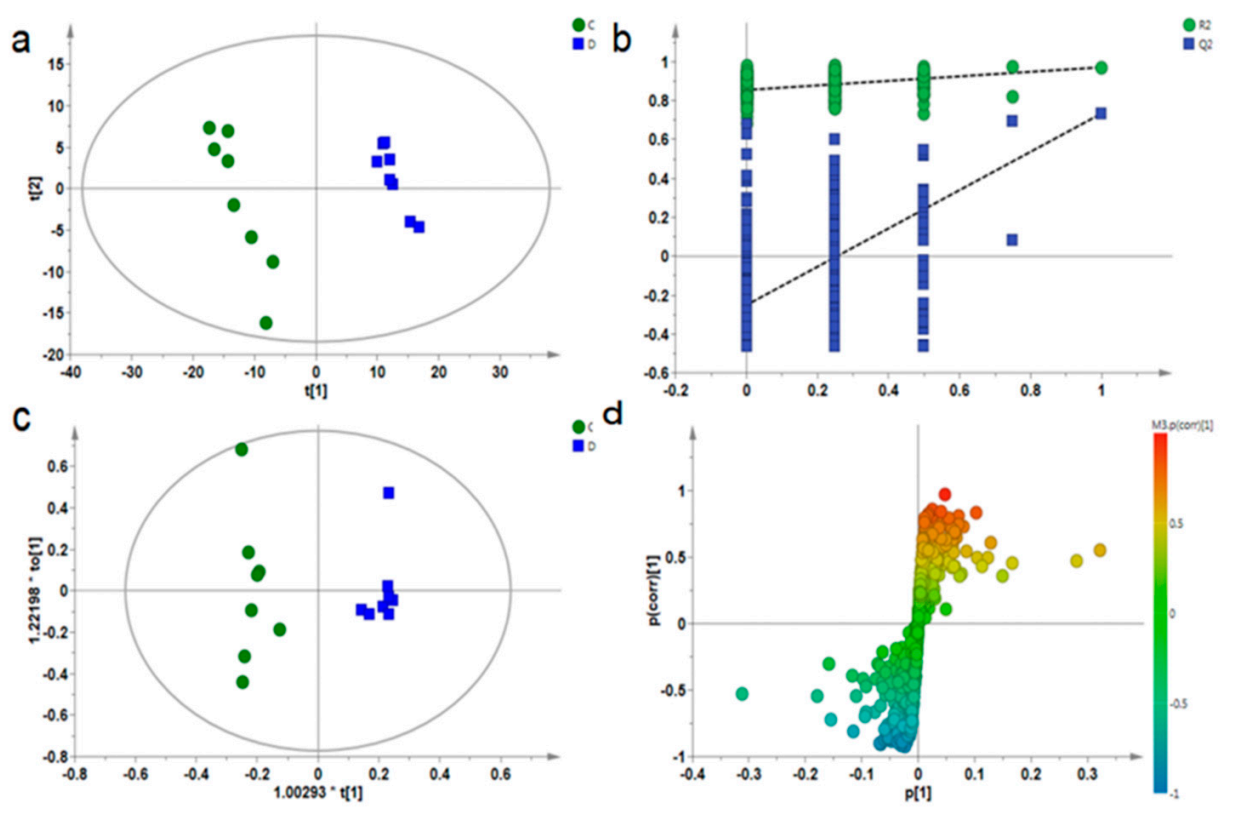

Figure 4. PLS-DA diagram (a), PLS-DA model verification diagram (b), OPLS-DA diagram (c), and corresponding S-plot diagram (d) for the gastrocnemius muscle samples of groups C and D.

a

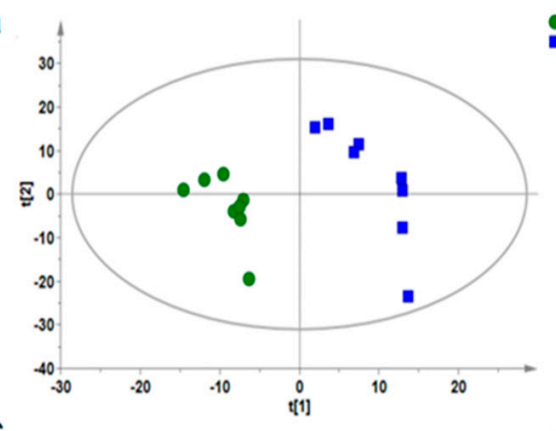

C

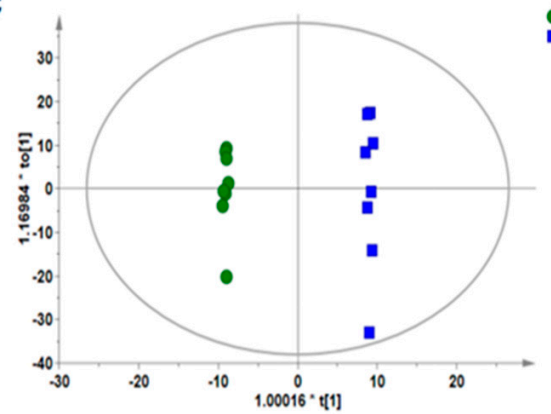

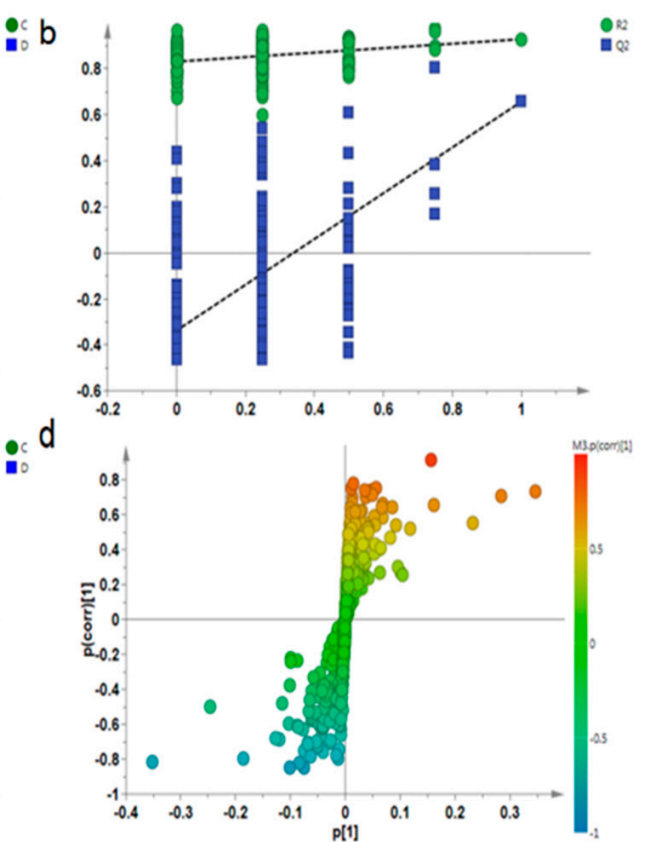

Figure 5. PLS-DA diagram (a), PLS-DA model verification diagram (b), OPLS-DA diagram (c), and corresponding S-plot diagram (d) for the soleus muscle samples of groups C and D.

As shown in Tables 1 and 2, the levels of propylene glycol, lactate, alanine, and alphaglucose in the gastrocnemius of group D were significantly increased compared to group C, while the levels of citric acid, lysine, creatinine, anserine, taurine, and choline phosphate were significantly reduced. After the endurance exercise intervention, five metabolites were significantly changed: the levels of propylene glycol, lactate, and alpha-glucose were decreased, and the levels of anserine and choline phosphate were increased. After the resistance exercise intervention, three metabolites were significantly changed: the level of propylene glycol was decreased, and the levels of lysine and citric acid were increased. 
Table 1. Ten metabolites and their trends of change in the gastrocnemius muscle.

\begin{tabular}{|c|c|c|c|c|c|c|}
\hline Number & $\begin{array}{c}\text { Potential } \\
\text { Biomarkers }\end{array}$ & Chemical Shift ( $\delta$ ) & VIP & D vs. C & E vs. D & R vs. D \\
\hline 1 & Propylene glycol & $1.25(\mathrm{~s})$ & 1.08 & $\uparrow *$ & $\downarrow$ & $\downarrow^{\#}$ \\
\hline 2 & Lactate & $1.33,4.12(\mathrm{~d})$ & 3.79 & $\uparrow *$ & $\downarrow$ & $\downarrow$ \\
\hline 3 & Lysine & $1.45,1.72,1.90(\mathrm{~s})$ & 1.1 & $\downarrow *$ & $\downarrow$ & $\uparrow \#$ \\
\hline 4 & Alanine & $1.49(\mathrm{~d})$ & 1.13 & $\uparrow * *$ & $\downarrow$ & $\downarrow$ \\
\hline 5 & Citric acid & $2.67(\mathrm{~s})$ & 1.29 & $\downarrow^{* *}$ & $\uparrow$ & $\uparrow \# \#$ \\
\hline 6 & Anserine & $3.02,4.52(\mathrm{~s})$ & 1.3 & $\downarrow *$ & $\uparrow \# \#$ & $\uparrow$ \\
\hline 7 & Creatinine & $3.05,3.92(\mathrm{~s})$ & 2.59 & $\downarrow *$ & $\uparrow$ & $\uparrow$ \\
\hline 8 & Choline phosphate & $3.21(\mathrm{~m})$ & 1.25 & $\downarrow *$ & $\uparrow \#$ & $\uparrow$ \\
\hline 9 & Taurine & $3.28(\mathrm{~s})$ & 2.36 & $\downarrow *$ & $\uparrow$ & $\uparrow$ \\
\hline 10 & Alpha-glucose & $3.90,5.25(\mathrm{~m})$ & 1.3 & $\uparrow * *$ & $\downarrow$ & $\downarrow$ \\
\hline
\end{tabular}

Table 2. Thirteen metabolites and their trends of change in the soleus muscle.

\begin{tabular}{|c|c|c|c|c|c|c|}
\hline Number & $\begin{array}{c}\text { Potential } \\
\text { Biomarkers }\end{array}$ & Chemical Shift ( $\delta$ ) & VIP & D vs. C & E vs. D & R vs. D \\
\hline 1 & Lactate & $1.35(\mathrm{~d})$ & 4.08 & $\downarrow^{* *}$ & $\uparrow \# \#$ & $\uparrow \#$ \\
\hline 2 & Alanine & $1.50(\mathrm{~d})$ & 1.81 & $\uparrow *$ & $\downarrow$ & $\downarrow$ \\
\hline 3 & Anserine & $3.02,7.08,8.1(\mathrm{~s})$ & 1.9 & $\downarrow^{*}$ & $\uparrow \#$ & $\uparrow$ \\
\hline 4 & Creatinine & $3.05,3.92(\mathrm{~s})$ & 4.01 & $\uparrow *$ & $\downarrow^{\# \#}$ & $\downarrow^{\#}$ \\
\hline 5 & Choline & $3.20(\mathrm{t})$ & 1.1 & $\downarrow^{*}$ & $\uparrow \#$ & $\uparrow$ \\
\hline 6 & Choline phosphate & $3.21(\mathrm{~m})$ & 1.77 & $\downarrow *$ & $\uparrow \#$ & $\uparrow$ \\
\hline 7 & $\begin{array}{l}\text { Glycerophosphate } \\
\text { choline }\end{array}$ & $3.23(\mathrm{~s})$ & 2.74 & $\downarrow^{*}$ & $\uparrow$ & $\uparrow$ \\
\hline 8 & Glycine & $3.56(\mathrm{~d})$ & 1.05 & $\uparrow *$ & $\downarrow$ & $\downarrow$ \#\# \\
\hline 9 & Glutamine & $3.77(\mathrm{~d})$ & 3.24 & $\downarrow^{* *}$ & $\uparrow$ & $\uparrow$ \\
\hline 10 & AMP & $4.02,6.15,8.25(\mathrm{t})$ & 1.35 & $\downarrow^{* *}$ & $\uparrow$ & $\downarrow$ \\
\hline 11 & Histidine & $7.04,7.11(\mathrm{~s})$ & 1.75 & $\downarrow^{* *}$ & $\uparrow \# \#$ & $\downarrow^{\#}$ \\
\hline 12 & Hypoxanthine & $8.13,8.21(\mathrm{~s})$ & 2.16 & $\uparrow * *$ & $\downarrow$ & $\uparrow$ \\
\hline 13 & Inosine & $8.24(\mathrm{~d})$ & 1.08 & $\downarrow *$ & $\uparrow \# \#$ & $\uparrow$ \\
\hline
\end{tabular}

D vs. C: ${ }^{*} p<0.05,{ }^{* *} p<0.01$; E vs. D, R vs. D: ${ }^{\#} p<0.05,{ }^{\# \#} p<0.01$; $\uparrow:$ Increase, $\downarrow$ : Decrease.

Compared with group $C$, the levels of alanine, creatinine, hypoxanthine, and glycine in the soleus muscle of group D were significantly increased, and the levels of lactate, choline, anserine, glycerophosphate choline, choline phosphate, inosine, glutamine, AMP, and histidine were significantly reduced. After the endurance exercise intervention, eight metabolites were significantly recalled: the levels of alanine and creatinine were decreased, while the levels of lactate, anserine, choline, choline phosphate, histidine, and inosine were increased. After the resistance exercise intervention, five metabolites were significantly changed: the levels of alanine, creatinine, glycine and histidine were decreased while the level of lactate was increased.

\subsubsection{Pathway Analysis}

The metabolites were imported into the MetaboAnalyst 4.0 (http:/ / www.metaboanalyst.ca (accessed on 25 November 2020)) for metabolic pathway enrichment analysis (Figures $6 \mathrm{~b}$ and $7 \mathrm{~b}$ ). On the horizontal axis, the larger the fold number, the more metabolites participated in the metabolic pathways. On the vertical axis, the darker (redder) the color, the more obvious the changes in metabolic pathways [24]. Therefore, MetPA analysis can explain the extent to which the metabolites or metabolic pathways have an impact on body metabolism. Moreover, MetPA analysis results (Figures6a,c and 7a,c) were consistent with enrichment analysis results. Holm $p$-value, FDR (False Discovery Rate), and Impact value were integrated to find that rats in group D regulated gastrocnemius muscle metabolism mainly through eight metabolic pathways (Figure 6a), including that of the glycolysis or gluconeogenesis, pyruvate metabolism, citrate 
acid (TCA) cycle, aminoacyl-tRNA biosynthesis, alanine-aspartate-glutamate metabolism, lysine biosynthesis, lysine degradation, and taurine-hypotaurine metabolism. Changes were observed in metabolite levels of various metabolic pathways such as glycolysis or gluconeogenesis, pyruvate metabolism, glycerophospholipid metabolism, glycerolipid metabolism, and starch-sucrose metabolism, in the gastrocnemius after endurance exercise (Figure 6c). Resistance exercise mainly improved the stress by regulating the metabolic pathways of pyruvate metabolism, TCA cycle, glycerolipid metabolism, aminoacyl-TRNA biosynthesis, lysine biosynthesis, and lysine degradation, in the gastrocnemius (Figure 6d).

\section{a}

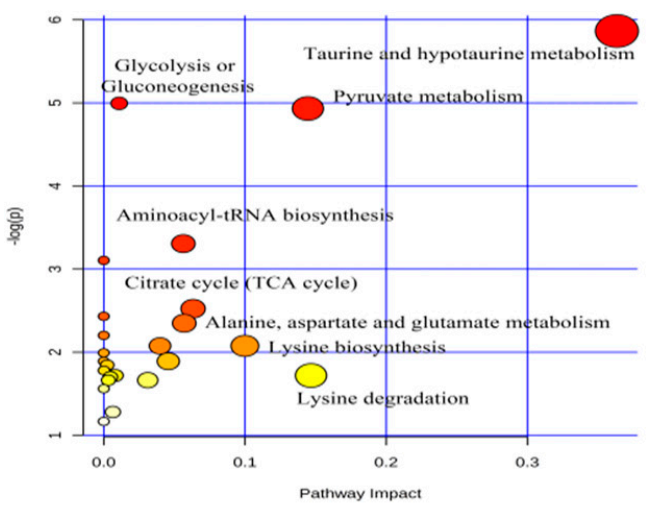

C

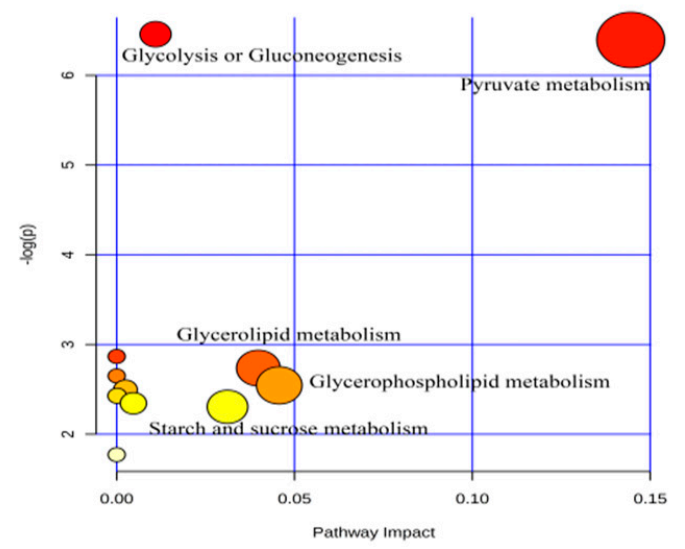

b

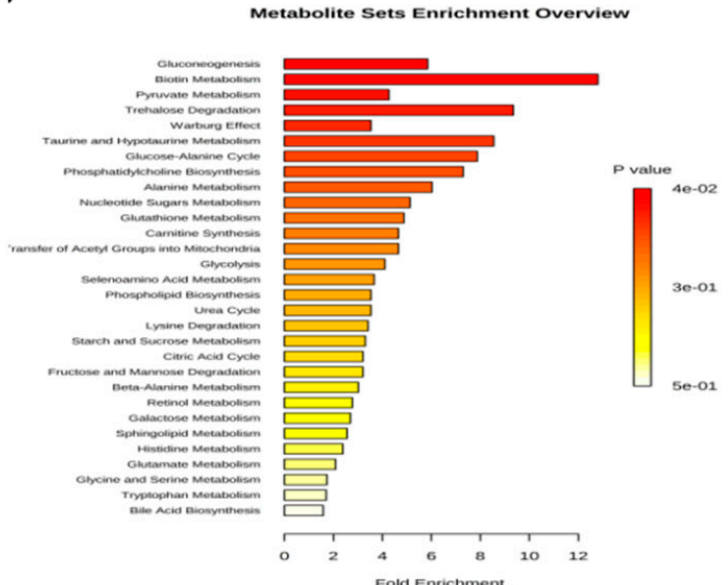

d

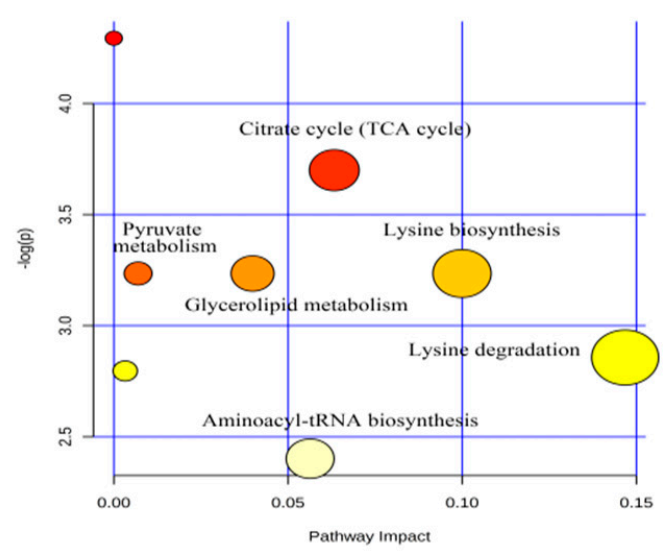

Figure 6. MetPA analysis of metabolic pathway ((a) C vs. D; (c) D vs. E; (d) D vs. R) and enrichment of metabolite sets in rat gastrocnemius muscle $(\mathbf{b})$.

The results from group D showed that there were also eight pathways were involved in the regulation of metabolism in the soleus, including that of the pyruvate metabolism, alanine-aspartate-glutamate metabolism, glycerophospholipid metabolism, glycine-serinethreonine metabolism, histidine metabolism, purine metabolism, D-glutamine-D-glutamate metabolism, and taurine and hypotaurine metabolism (Figure 7a). The improved response to stress by endurance exercise appeared to be mainly associated with the changes in the metabolic pathways of the pyruvate metabolism, alanine-aspartate-glutamate metabolism, glycerophospholipid metabolism, histidine metabolism, taurine and hypotaurine metabolism, in the soleus muscle (Figure 7c). The resistance exercise was shown to have mainly affected the metabolic pathways of pyruvate metabolism, alanine-aspartate-glutamate metabolism, glycine-serine-threonine metabolism, histidine metabolism, and taurine and hypotaurine metabolism, in the soleus, that appeared to be associated with the improvement in the symptoms of depression. 
a

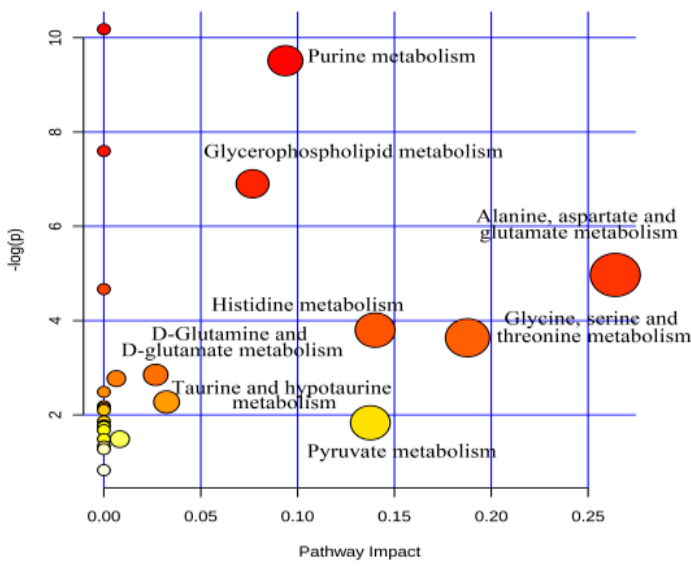

C

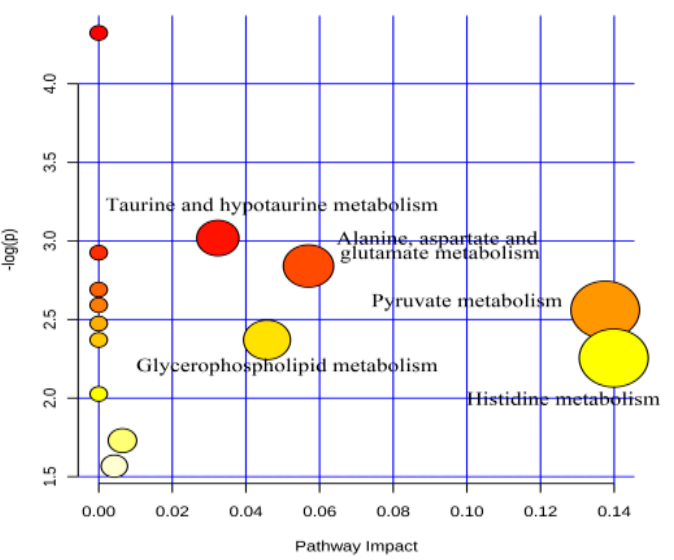

b

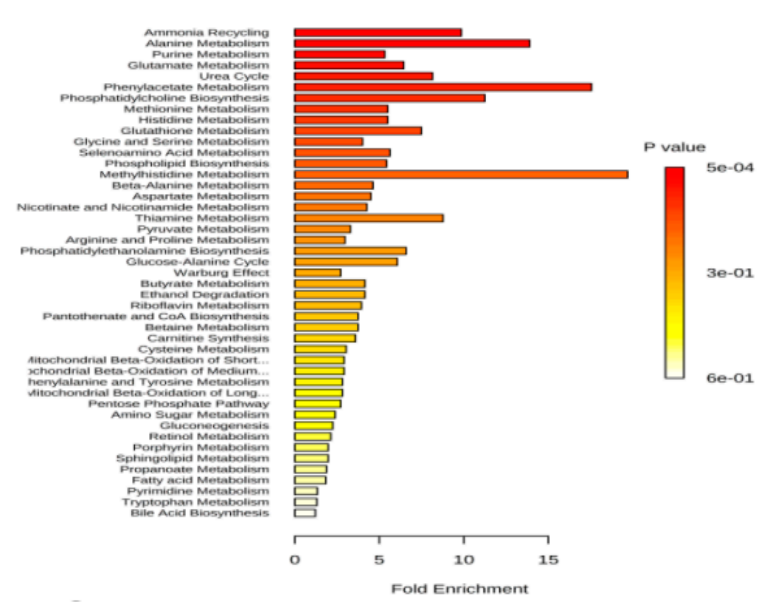

d

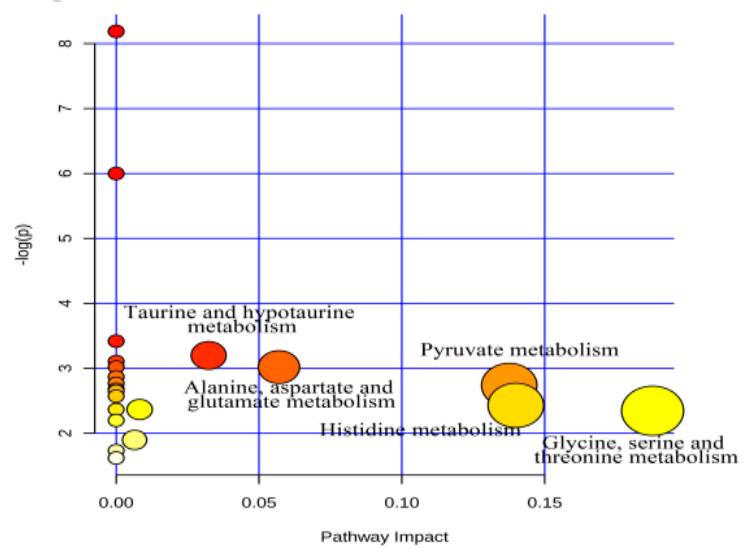

Figure 7. MetPA analysis of metabolic pathway ((a) C vs. D; (c) D vs. E; (d) D vs. R) and enrichment of metabolite sets in rat soleus muscle (b).

\section{Discussion}

The results of this study showed that both the endurance (group E) and resistance (group R) exercise interventions had affected the responses to stress as compared with the untreated rats with depression-like symptoms (group D) (Figure 2). Three behavioral indicators (the sucrose preference rate and the numbers of crossings and standing) in group $\mathrm{E}$ were significantly higher than those in group $\mathrm{D}$. However, only the number of crossing in group $\mathrm{R}$ was significantly higher than that in group $\mathrm{D}$. These results may indicate that the exercise interventions can effectively alleviate the stress in rats, and the endurance exercise appears to have broader effects than the resistance exercise.

\subsection{Effects of Endurance and Resistance Exercise on Differential Metabolites in the Gastrocnemius Muscle}

The gastrocnemius muscle mainly consists of fast-twitch fibers, which are physiologically characterized by higher contraction speed, muscle tension, fatiguability, enzyme activities of anaerobic metabolism [25], and larger glycogen reserves [26], as compared with slow-twitch muscle fibers. The significant changes of several metabolites in the gastrocnemius are worthy of further comments.

Lactate is a by-product of anaerobic glycolysis. It was found that the level of lactate in group D was higher than that in group $C$, indicating that the anaerobic glycolysis in the gastrocnemius of the rats under CUMS was increased, or the ability of muscle cells to clear lactate was decreased. Creatinine is a metabolite of creatine phosphate and an important energy storage substance in skeletal muscle. The decreased creatinine level in group D suggests that there may be abnormalities in the creatine phosphate energy supply system 
in the gastrocnemius of the rats under CUMS. Similarly, energy metabolism disorders have been found to be common in animal models of depression [27]. In our study, the four weeks of endurance exercise significantly reversed the CUMS-induced change in lactate in the gastrocnemius (Table 1), suggesting that the improved responses to stress by the endurance exercise could be related to the changes in glycolysis.

Citric acid as an antioxidant can effectively reduce oxidative stress in the body and promote metabolism through the TCA cycle [28]. At the same time, it is related to the secretion of various hormones associated with depression, such as corticotropic hormone, epinephrine, and norepinephrine [29]. Therefore, citrate may be a key mood "regulator" [30]. In this study, the CUMS resulted in a significant decrease in the level of citric acid and an abnormal increase in alpha-glucose and alanine in the gastrocnemius muscle, indicating impaired TCA cycling metabolism in muscle cells. After the four weeks of exercise, the citric acid level was significantly increased in the R group (Table 1), suggesting that resistance exercise could have improved the TCA cycle in the gastrocnemius muscle.

Taurine has a protective effect on lipid peroxidation of the cell membrane [31]. Lack of lysine and taurine in the muscle may lead to tissue damage and inflammation [32], and inflammation was one of the important factors causing depression. Compared with group $\mathrm{D}$ of this study, the level of lysine in the gastrocnemius of the R group was significantly increased. Although the taurine level did not change significantly, it showed a trend of callback (Table 1). It can be suggested that the resistance exercise played an antidepressant role by regulating lysine metabolism. However, there were no significant changes in lysine and taurine levels in the gastrocnemius muscle of group E, suggesting the specific effect of the resistance exercise on this pathway.

It has been reported that propylene glycol is closely associated with early central nervous system disorders in humans, such as depression, epilepsy, and other diseases [33]. Recently, there has been a report on the toxicity of propylene glycol in young and adult human bodies, which can induce apoptosis in the developing central nervous system [34]. In this study, propylene glycol in the gastrocnemius of group D was significantly higher than that of group C (Table 1). It can be speculated that the propylene glycol from skeletal muscle may exert toxic effects on the central nervous system, although this needs to be confirmed by further investigation. Compared with group D, groups E and R showed significant callback of propylene glycol, suggesting that exercise can alleviate the weakly toxic effect of propylene glycol in skeletal muscle of the rats with CUMS, and play a positive role in improving responses to stress.

Anserine is an endogenous dipeptide composed of histidine (or the methylated form of histidine) and alanine, which is found in the skeletal muscle of vertebrates [35]. Anserine and its analogs (carnosine and homocarnosine) are small molecules and water-soluble. Complement therapy with anserine and carnosine has been reported to be effective in improving cognitive impairment in the elderly [36]. Our results showed that the anserine in the gastrocnemius muscle of the rats under CUMS was significantly decreased, while it was significantly increased after four weeks of endurance exercise, suggesting that endurance exercise could improve stress by regulating the histidine metabolism pathway.

\subsection{Effects of Endurance and Resistance Exercise on Differential Metabolites in the Soleus Muscle}

It is known that the soleus, as a typical slow-twitch muscle, has different metabolic characteristics compared to the fast-twitch gastrocnemius [37]. However, the effects of exercise interventions on depression in relation to the metabolic characteristics of different types of muscle fibers have not been reported previously. Some of the specific metabolomic changes in the soleus in response to the exercise interventions are highlighted below.

Glutamate is an excitatory neurotransmitter, which has been proved to be closely related to the incidence of depression [38]. Glutamate plays an important role in the synthesis of glutamine, and the phosphorylated glutamine enzyme can hydrolyze glutamine to glutamate. The reduction of glutamine found in the soleus muscle of group D might be due to its conversion to more glutamate, however, this is contrary to the results of another 
study on the cerebrospinal fluid [39]. So, the specific mechanism needs to be further investigated. Pyruvate and glutamate can be catalyzed by alanine aminotransferase (ALT) to form alanine and 2-oxoglutarate [40], which plays an important role in gluconeogenesis and amino acid metabolism. Therefore, an increase in glutamate may lead to an increase in alanine content. In this study, the level of glutamate did not change significantly, while the level of glutamine was significantly decreased and the level of alanine was significantly increased in group $\mathrm{D}$. The levels of alanine in soleus muscle of rats in groups $\mathrm{E}$ and $\mathrm{R}$ were significantly decreased compared with those in group $\mathrm{D}$, suggesting that exercise might improve stress by regulating alanine-aspartate-glutamate metabolic pathways.

A recent study showed that exercise-induced endogenous lactate crossed the bloodbrain barrier and increased PGC-1 and FNDC5 levels by activating SIRT1, which in turn enhanced BDNF signaling in the hippocampus, thereby facilitating learning and memory [41]. We found that the level of lactate in the soleus muscle of the rats under CUMS was significantly reduced and that in the groups $\mathrm{E}$ and $\mathrm{R}$ were significantly higher than that in group D, suggesting that exercise-induced improvement might be related to this pathway that might have affected the expression of BDNF in the hippocampus. Interestingly, the changes of lactate in the gastrocnemius and soleus were not the same, therefore, the above speculation would require further study.

Glycine is an amino acid that can be used as a substrate in gluconeogenesis and an important energy storage substance in the body. It has the effect of improving metabolic disorders [42] and is involved in the regulation of the inflammatory response in the recovery process after muscle injury [43]. The results of this study showed that the glycine level in the soleus muscle of group D was significantly higher than that in group $\mathrm{C}$, and that in group $\mathrm{R}$ was significantly lower compared to group D (Table 2). It can be speculated that there was an inflammatory response in the soleus muscle under CUMS, and glycine was increased as an anti-inflammatory substance to improve the body's response to stress. However, it has also been reported that glycine level was decreased in depression, and glycine might not play a protective role in tissues when some cells were dying [44]. Therefore, further studies are required to examine the relationship between muscle glycine level and depression.

It has been reported that people with major depression show significantly lower levels of polyunsaturated fatty acids and inosine, and polyunsaturated fatty acids may play a role in the pathophysiology of depression by regulating the purine metabolic pathway [45]. Supplementation of hypoxanthine could repair the dysfunction of cells and improve the pathological state of the body [46]. Compared with group C, hypoxanthine in the soleus muscle of group $\mathrm{D}$ was significantly higher, while inosine was significantly lower (Table 2). The endurance exercise appeared to be able to increase the level of inosine in the soleus significantly.

Glycerophosphate choline can cross the blood-brain barrier and provide essential choline for the synthesis of phosphorylcholine and acetylcholine. It plays a neuroprotective role through anti-inflammatory effects [47], which is conducive to the improvement of learning, memory, and cognitive functions [48]. Choline is an important component of biofilms, which can regulate cell apoptosis and promote fat metabolism. Moreover, choline is a precursor of acetylcholine, which is an important neurotransmitter in the central cholinergic system and is closely related to learning and cognitive activities [49]. It has been reported that the plasma choline level decreased in patients with depression [50]. Our results showed that the contents of glycerophosphate choline, choline phosphate, and choline in the soleus muscle of the rats under CUMS were significantly decreased, suggesting a disorder of intracellular glycerophosphate choline metabolism. In response to the endurance exercise, both choline phosphate and choline in group E showed a significant callback, indicating that the improved response to the stress might be related to the changes in the choline metabolism.

In summary, we identified for the first time significant changes of 10 differential metabolites in the gastrocnemius muscle of the rats under CUMS. These metabolites were mainly involved in the TCA cycle, glucose metabolism, and phosphocreatine metabolism. The endurance and resistance exercise as prescribed in this study could recall the changes 
in five and three different metabolites, respectively (Table 1). We also identified 13 differential metabolites in the soleus muscle, which were mainly involved in lipid and choline metabolism. The endurance and resistance exercise could recall the changes in seven and five different metabolites, respectively (Table 2). Overall, the endurance exercise appeared to have more extensive effects on metabolites in both gastrocnemius and soleus.

The limitations of the study may include that, first, due to the constraints of time and resources, we could only deliver four weeks of exercise intervention. Whether a longer term of CUMS and exercise intervention would result in different anti-depression effects is unknown. However, it appears that it would require a minimum of four weeks of exercise training to obtain statistically significant changes in the behavioral indices, as no significant changes were found in the first three weeks of exercise intervention (Figure 2). Secondly, only one level of exercise intensity and volume was used for either the endurance or resistance exercise, therefore, the dose-response relationship for the intervention effects could not be established in this study. Thirdly, although the soleus and gastrocnemius are typical slow oxidative and fast glycolytic muscles in rodents, the effects of exercise interventions on other muscles (e.g., with mixed slow and fast fibers) cannot be predicted from the current results.

\section{Conclusions}

The outcomes of this study indicated that both the endurance and resistance exercise as prescribed could improve behavioral indices of depression in rats under CUMS, with the endurance exercise appeared to have a better effect. The metabolomic analysis identified significant changes in 10 metabolites from the gastrocnemius and 13 metabolites from the soleus muscle in rats under CUMS compared to the blank control, suggesting an association between the metabolic profile and depression. Both types of exercise intervention had a callback effect on the changes in several metabolites (five and eight in group $E$ and three and five in group $\mathrm{R}$, in the gastrocnemius and soleus, respectively), indicating that a number of metabolic pathways in skeletal muscle were involved in the improved responses to stress, and the endurance exercise might have a broader effect.

Supplementary Materials: The following are available online at https:/ /www.mdpi.com/1660-4 601/18/4/1645/s1, Table S1: Changes in the body weight (g) of rats in different groups during the experiment, Table S2: Changes in the sucrose preference (\%) of rats in different groups during the experiment, Table S3: Changes in the number of standing of rats in different groups during the experiment, Table S4: Changes in the number of crossing of rats in different groups during the experiment.

Author Contributions: Conceptualization, Y.H. and J.T.; validation, X.Q. and A.C.; investigation, Y.H., X.L. and X.X.; supervision, S.Z. and X.Q.; writing—original draft, Y.H. and X.X.; writingreview and editing, X.L. and S.Z. All authors have read and agreed to the published version of the manuscript.

Funding: This research was supported by the NSFC (National Natural Science Foundation of China, grant: 82074147) and Key R\&D projects of Shanxi Province, China (grant: 201903D321022).

Institutional Review Board Statement: The study was conducted according to the guidelines of the Declaration of Helsinki, and approved by the Institutional Review Board (or Ethics Committee) of the Shanxi University (protocol code: SXULL2018006, date of approval: 6 March 2018).

Informed Consent Statement: Not applicable.

Data Availability Statement: Data is contained within the article or supplementary material. The data presented in this study are available in [insert article or supplementary material here].

Conflicts of Interest: The authors declared no conflict of interest. 


\section{References}

1. GBD 2017 Disease and Injury Incidence and Prevalence Collaborators. Global, regional, and national incidence, prevalence, and years lived with disability for 354 diseases and injuries for 195 countries and territories, 1990-2017: A systematic analy-sis for the Global Burden of Disease Study 2017. Lancet 2018, 392, 1789-1858. [CrossRef]

2. WHO Fact Sheet on Depression. Available online: https://www.who.int/news-room/fact-sheets/detail/depression (accessed on 25 November 2020).

3. Dean, J.; Keshavan, M. The neurobiology of depression: An integrated view. Asian J. Psychiatry 2017, 27, 101-111. [CrossRef]

4. Chan, K.L.; Cathomas, F.; Russo, S.J. Central and Peripheral Inflammation Link Metabolic Syndrome and Major Depressive Disorder. Physiology 2019, 34, 123-133. [CrossRef]

5. Dunn, A.L.; Trivedi, M.H.; Kampert, J.B.; Clark, C.G.; Chambliss, H.O. Exercise treatment for depression. Am. J. Prev. Med. 2005, 28, 1-8. [CrossRef]

6. Perraton, L.G.; Kumar, S.; Machotka, Z. Exercise parameters in the treatment of clinical depression: A systematic review of randomized controlled trials. J. Eval. Clin. Pract. 2010, 16, 597-604. [CrossRef]

7. Stanton, R.; Reaburn, P. Exercise and the treatment of depression: A review of the exercise program variables. J. Sci. Med. Sport 2014, 17, 177-182. [CrossRef]

8. Fournier, N.M.; Duman, R.S. Role of vascular endothelial growth factor in adult hippocampal neurogenesis: Implications for the pathophysiology and treatment of depression. Behav. Brain Res. 2012, 227, 440-449. [CrossRef]

9. Park, H.; Poo, M.-M. Neurotrophin regulation of neural circuit development and function. Nat. Rev. Neurosci. 2013, 14, 7-23. [CrossRef]

10. Yau, S.Y.; Li, A.; Hoo, R.L.C.; Ching, Y.P.; Christie, B.R.; Lee, T.M.C.; Xu, A.; So, K.-F. Physical exercise-induced hippocampal neurogenesis and antidepressant effects are mediated by the adipocyte hormone adiponectin. Proc. Natl. Acad. Sci. USA 2014, 111, 15810-15815. [CrossRef] [PubMed]

11. Guijas, C.; Montenegro-Burke, J.R.; Warth, B.; E Spilker, M.; Siuzdak, G. Metabolomics activity screening for identifying metabolites that modulate phenotype. Nat. Biotechnol. 2018, 36, 316-320. [CrossRef]

12. Quinones, M.P.; Kaddurah-Daouk, R. Metabolomics tools for identifying biomarkers for neuropsychiatric diseases. Neurobiol. Dis. 2009, 35, 165-176. [CrossRef]

13. Fiorenza, M.; Gunnarsson, T.P.; Hostrup, M.; Iaia, F.M.; Schena, F.; Pilegaard, H.; Bangsbo, J. Metabolic stress-dependent regulation of the mitochondrial biogenic molecular response to high-intensity exercise in human skeletal muscle. J. Physiol. 2018, 596, 2823-2840. [CrossRef]

14. Marzetti, E.; on behalf of the SPRINTT Consortium; Calvani, R.; Tosato, M.; Cesari, M.; Di Bari, M.; Cherubini, A.; Broccatelli, M.; Savera, G.; D'Elia, M.; et al. Physical activity and exercise as countermeasures to physical frailty and sarcopenia. Aging Clin. Exp. Res. 2017, 29, 35-42. [CrossRef]

15. Uchitomi, R.; Hatazawa, Y.; Senoo, N.; Yoshioka, K.; Fujita, M.; Shimizu, T.; Miura, S.; Ono, Y.; Kamei, Y. Metabolomic Analysis of Skeletal Muscle in Aged Mice. Sci. Rep. 2019, 9, 1-11. [CrossRef]

16. Tian, J.-S.; Shi, B.-Y.; Xiang, H.; Gao, S.; Qin, X.-M.; Du, G.-H. 1H-NMR-Based Metabonomic Studies on the Anti-Depressant Effect of Genipin in the Chronic Unpredictable Mild Stress Rat Model. PLoS ONE 2013, 8, e75721. [CrossRef]

17. Legrand, F.; Neff, E.M. Efficacy of exercise as an adjunct treatment for clinically depressed inpatients during the initial stages of antidepressant pharmacotherapy: An open randomized controlled trial. J. Affect. Disord. 2016, 191, 139-144. [CrossRef]

18. Schlittler, M.; Goiny, M.; Agudelo, L.Z.; Venckunas, T.; Brazaitis, M.; Skurvydas, A.; Kamandulis, S.; Ruas, J.L.; Erhardt, S.; Westerblad, H.; et al. Endurance exercise increases skeletal muscle kynurenine aminotransferases and plasma kynurenic acid in humans. Am. J. Physiol. Physiol. 2016, 310, C836-C840. [CrossRef] [PubMed]

19. Stricker, P.R.; Faigenbaum, A.D.; McCambridge, T.M. COUNCIL ON SPORTS MEDICINE AND FITNESS Resistance Training for Children and Adolescents. Pediatrics 2020, 145, e20201011. [CrossRef] [PubMed]

20. Fragala, M.S.; Cadore, E.L.; Dorgo, S.; Izquierdo, M.; Kraemer, W.J.; Peterson, M.D.; Ryan, E.D. Resistance Training for Older Adults. J. Strength Cond. Res. 2019, 33, 2019-2052. [CrossRef] [PubMed]

21. Han, Y.; Jia, Y.; Tian, J.; Zhou, S.; Chen, A.; Luo, X. Urine metabolomic responses to aerobic and resistance training in rats under chronic unpredictable mild stress. PLoS ONE 2020, 15, e237377. [CrossRef]

22. Malatynska, E.; Steinbusch, H.W.M.; Redkozubova, O.; Bolkunov, A.; Kubatiev, A.; Yeritsyan, N.B.; Vignisse, J.; Bachurin, S.; Strekalova, T. Anhedonic-like traits and lack of affective deficits in 18-month-old C57BL/6 mice: Implications for modeling elderly depression. Exp. Gerontol. 2012, 47, 552-564. [CrossRef] [PubMed]

23. Wang, Q.; Timberlake, M.A.; Prall, K.; Dwivedi, Y. The recent progress in animal models of depression. Prog. Neuro-Psychopharmacol. Biol. Psychiatry 2017, 77, 99-109. [CrossRef]

24. Xia, J.; Wishart, D.S. MSEA: A web-based tool to identify biologically meaningful patterns in quantitative metabolomic data. Nucleic Acids Res. 2010, 38, W71-W77. [CrossRef]

25. Akmali, A.; Saghebjoo, M. High-intensity interval training with long duration intervals is more effective than short duration intervals for improving glycolytic capacity in the rats' gastrocnemius muscle. Horm. Mol. Biol. Clin. Investig. 2019, 41. [CrossRef]

26. Phua, W.W.T.; Wong, M.X.Y.; Liao, Z.; Tan, N.S. An aPPARent Functional Consequence in Skeletal Muscle Physiology via Peroxisome Proliferator-Activated Receptors. Int. J. Mol. Sci. 2018, 19, 1425. [CrossRef] [PubMed] 
27. Réus, G.Z.; Nacif, M.P.; Abelaira, H.M.; Tomaz, D.B.; Dos Santos, M.A.B.; Carlessi, A.S.; I Matias, B.; Da Luz, J.R.; Steckert, A.V.; Jeremias, G.C.; et al. Ketamine Treatment Partly Reverses Alterations in Brain Derived- Neurotrophic Factor, Oxidative Stress and Energy Metabolism Parameters Induced by an Animal Model of Depression. Curr. Neurovasc. Res. 2015, 12, 73-84. [CrossRef]

28. Akram, M. Citric Acid Cycle and Role of its Intermediates in Metabolism. Cell Biophys. 2014, 68, 475-478. [CrossRef]

29. Setoyama, D.; Kato, T.A.; Hashimoto, R.; Kunugi, H.; Hattori, K.; Hayakawa, K.; Sato-Kasai, M.; Shimokawa, N.; Kaneko, S.; Yoshida, S.; et al. Plasma Metabolites Predict Severity of Depression and Suicidal Ideation in Psychiatric Patients-A Multicenter Pilot Analysis. PLoS ONE 2016, 11, e0165267. [CrossRef]

30. Yoshimi, N.; Futamura, T.; Kakumoto, K.; Salehi, A.M.; Sellgren, C.M.; Holmén-Larsson, J.; Jakobsson, J.; Pålsson, E.; Landén, M.; Hashimoto, K. Blood metabolomics analysis identifies abnormalities in the citric acid cycle, urea cycle, and amino acid metabolism in bipolar disorder. BBA Clin. 2016, 5, 151-158. [CrossRef] [PubMed]

31. Jiang, L.; Huang, J.; Wang, Y.; Tang, H. Metabonomic Analysis Reveals the CCl4-Induced Systems Alterations for Multiple Rat Organs. J. Proteome Res. 2012, 11, 3848-3859. [CrossRef]

32. Dort, J.; Leblanc, N.; Maltais-Giguère, J.; Liaset, B.; Côté, C.H.; Jacques, H. Beneficial Effects of Cod Protein on Inflammatory Cell Accumulation in Rat Skeletal Muscle after Injury Are Driven by Its High Levels of Arginine, Glycine, Taurine and Lysine. PLoS ONE 2013, 8, e77274. [CrossRef]

33. Arulanantham, K.; Genel, M. Central nervous system toxicity associated with ingestion of propylene glycol. J. Pediatr. 1978, 93, 515-516. [CrossRef]

34. Lau, K.; Swiney, B.S.; Reeves, N.; Noguchi, K.K.; Farber, N.B. Propylene glycol produces excessive apoptosis in the developing mouse brain, alone and in combination with phenobarbital. Pediatr. Res. 2011, 71, 54-62. [CrossRef]

35. Derave, W.; De Courten, B.; Baba, S.P. An update on carnosine and anserine research. Amino Acids 2019, 51, 1-4. [CrossRef]

36. Kawahara, M.; Tanaka, K.-I.; Kato-Negishi, M. Zinc, Carnosine, and Neurodegenerative Diseases. Nutrients 2018, 10 , 147. [CrossRef]

37. Schiaffino, S.; Reggiani, C. Fiber Types in Mammalian Skeletal Muscles. Physiol. Rev. 2011, 91, 1447-1531. [CrossRef]

38. Lener, M.S.; Niciu, M.J.; Ballard, E.D.; Park, M.; Park, L.T.; Nugent, A.C.; Zarate, C.A. Glutamate and Gamma-Aminobutyric Acid Systems in the Pathophysiology of Major Depression and Antidepressant Response to Ketamine. Biol. Psychiatry 2017, 81, 886-897. [CrossRef]

39. Hashimoto, K.; Bruno, D.; Nierenberg, J.; Marmar, C.R.; Zetterberg, H.; Blennow, K.; Pomara, N. Abnormality in glutamineglutamate cycle in the cerebrospinal fluid of cognitively intact elderly individuals with major depressive disorder: A 3-year follow-up study. Transl. Psychiatry 2016, 6, e744. [CrossRef]

40. Frank, D.; Kuts, R.; Tsenter, P.; Gruenbaum, B.F.; Grinshpun, Y.; Zvenigorodsky, V.; Shelef, I.; Natanel, D.; Brotfain, E.; Zlotnik, A.; et al. The effect of pyruvate on the development and progression of post-stroke depression: A new therapeutic approach. Neuropharmacology 2019, 155, 173-184. [CrossRef]

41. El Hayek, L.; Khalifeh, M.; Zibara, V.; Abi Assaad, R.; Emmanuel, N.; Karnib, N.; El-Ghandour, R.; Nasrallah, P.; Bilen, M.; Ibrahim, P.; et al. Lactate mediates the effects of exercise on learning and memory through SIRT1-dependent activation of hippocampal brain-derived neurotrophic factor (BDNF). J. Neurosci. 2019, 39, 2369-2382. [CrossRef]

42. Razak, M.A.; Begum, P.S.; Viswanath, B.; Rajagopal, S. Multifarious Beneficial Effect of Nonessential Amino Acid, Glycine: A Review. Oxid. Med. Cell Longev. 2017, 2017, 1716701. [CrossRef] [PubMed]

43. Alves, A.; Bassot, A.; Bulteau, A.-L.; Pirola, L.; Morio, B. Glycine Metabolism and Its Alterations in Obesity and Metabolic Diseases. Nutrients 2019, 11, 1356. [CrossRef]

44. Weinberg, J.M.; Bienholz, A.; Venkatachalam, M.A. The role of glycine in regulated cell death. Cell. Mol. Life Sci. 2016, 73, 2285-2308. [CrossRef] [PubMed]

45. Zhou, X.; Liu, L.; Lan, X.; Cohen, D.; Zhang, Y.; Ravindran, A.V.; Yuan, S.; Zheng, P.; Coghill, D.; Yang, L.; et al. Polyunsaturated fatty acids metabolism, purine metabolism and inosine as potential independent diagnostic biomarkers for major depressive disorder in children and adolescents. Mol. Psychiatry 2019, 24, 1478-1488. [CrossRef]

46. Lee, J.S.; Wang, R.X.; Alexeev, E.E.; Lanis, J.M.; Battista, K.D.; Glover, L.E.; Colgan, S.P. Hypoxanthine is a checkpoint stress metabolite in colonic epithelial energy modulation and barrier function. J. Biol. Chem. 2018, 293, 6039-6051. [CrossRef] [PubMed]

47. Lee, S.H.; Choi, B.Y.; Kim, J.H.; Kho, A.; Sohn, M.; Song, H.K.; Choi, H.C.; Suh, S.W. Late treatment with choline alfoscerate (l-alpha glycerylphosphorylcholine, $\alpha$-GPC) increases hippocampal neurogenesis and provides protection against seizure-induced neuronal death and cognitive impairment. Brain Res. 2017, 1654, 66-76. [CrossRef]

48. Tayebati, S.K.; Martinelli, I.; Moruzzi, M.; Amenta, F.; Tomassoni, D. Choline and Choline alphoscerate Do Not Modulate Inflammatory Processes in the Rat Brain. Nutrients 2017, 9, 1084. [CrossRef] [PubMed]

49. Ren, J.; Ding, X.; Greer, J.J. Activating $\alpha 4 \beta 2$ Nicotinic Acetylcholine Receptors Alleviates Fentanyl-induced Respiratory Depression in Rats. Anesthesiology 2019, 130, 1017-1031. [CrossRef] [PubMed]

50. De Diego-Adeliño, J.; Portella, M.J.; Gomez-Anson, B.; López-Moruelo, O.; Serra-Blasco, M.; Vives-Gilabert, Y.; Puigdemont, L.; Pérez-Egea, R.; Álvarez, E.; Pérez, V. Hippocampal abnormalities of glutamate/glutamine, N-acetylaspartate and choline in patients with depression are related to past illness burden. J. Psychiatry Neurosci. 2013, 38, 107-116. [CrossRef] 